Improvements in dyspnoea were graded according to improvement in BORG score. An adverse effect of a headache was noted in one patient occurring 48 hours after nebulised morphine had commenced.

Conclusion Nebulised morphine and fentanyl have been used to improve dyspnoea due to $\mathrm{CF}$ in a very limited number of patients, therefore no conclusion advocating the use of nebulised opioids can be made. Further research in the form of randomised controlled trials is needed to improve the evidence base of symptom control in patients with CF. This research must also focus on adverse gastrointestinal side effects such as constipation increasing the risk of DIOS.

\section{RECOGNITION OF SEPSIS IN HOSPICE PATIENTS WITH FEVER}

Rachel Whitehorn. Douglas Macmillan Hospice

\subsection{6/bmjspcare-2019-ASP.181}

Background Historically, patients in hospices have not had routine physiological observations recorded, with the focus being on maintaining comfort at end of life. In recent years, more patients admitted to hospices still wish to receive active life-prolonging treatment, including for sepsis. NICE issued guidance on the recognition of possible sepsis in the community and hospital settings in summer 2016. Recognition includes assessment of patients' basic physiological observations.

This audit was performed to assess the extent to which basic physiological observations are completed to allow careful assessment of patients in the hospice for the presence of sepsis.

Assessment for sepsis risk includes

- Presence of risk factors

- altered conscious state,

- reduction in functional ability

- respiratory rate

- new need for oxygen to maintain saturations

- heart rate, blood pressure

- skin/lip/tongue colour

- temperature

- when the patient last passed urine

- Site of any likely infection.

Method retrospective review of patient records.

Results 15 episodes of pyrexia were identified in 11 patients in a four month period. Two of these were related to blood transfusion and not included in analysis. On assessment of the patients, blood pressure, heart rate, conscious state and functional level were the most frequently recorded sepsis risk factors. Skin colour, respiratory rate, presence of signs of skin or wound infection and urine output were hardly ever or never recorded as assessed.

Nursing staff requested medical assessment in all cases although this could be delayed until normal working hours. Intravenous antibiotics were started in two patients and oral antibiotics given in five. Ceiling of care was discussed in three cases.

Conclusion Decisions are made about the presence of sepsis and the use of antibiotics based on a limited patient assessment. Staff need to be reminded that fuller assessment of basic physiological observations may improve decisionmaking.

\section{OPIOID AUDIT - IN PURSUIT OF APPROPRIATE SYMPTOM MANAGEMENT}

Elizabeth Heard, Alison Franks. University Hospitals Coventry and Warwickshire NHS Trust

\subsection{6/bmjspcare-2019-ASP.182}

Background In a recent spate of referrals regarding pain control we found constipation was the major issue. This prompted us to question the commonality of this, as assessing and managing pain are core medical skills which, if done inadequately, can result in incorrect/inappropriate patient management.

Opioids, an important part of pain management, are widely used, by all doctors. The common side effects are well recognised. There are several different opioids, all of which can cause temporary nausea/vomiting and persistent issues with constipation. Prescribing errors are not uncommon. Thus we conducted an in-patient, Trust-wide spot audit of drug charts for prevalence of opioid prescribing, and anticipation of common side effects.

Method All adult care (non-ITU) wards were visited, drug charts reviewed and spreadsheet data collection tool populated.

Results In total, 782 charts were assessed - 440 (56\%) included an opioid.

Morphine was commonest, 55\%, codeine 32\%, oxycodone $4 \%$, fentanyl 3\%, buprenorphine, methadone and tramadol $2 \%$ each, dihydrocodeine $1 \%$, one prescription each for pethidine and alfentanil.

Of the prescriptions, 229 were for 'regular' opioids and 418 'as needed' - 28 (4\%) of these did not specify frequency of opioid use.

Bowel habit was recorded on $69 \%$ of the charts. At least one laxative was prescribed on 267 charts (61\%), totalling 450 prescriptions - senna 176 prescriptions, lactulose 144, macrogol 77, docusate 31 , glycerine suppositories 11 , six phosphate enemas and one for naloxegol.

Conclusions Appropriately used, opioids are invaluable for managing pain, but cause well recognised common side effects of which constipation can cause the greatest pain, distress and misery for our patients. It's relatively straightforward to manage, but needs to be anticipated, monitored for and addressed effectively to minimise patients suffering because we aren't questioning and recognising symptoms for what they are and prescribing correctly. More education planned.

\section{SURPRISING FINDINGS IN TRACKING PAIN PROGRESSION IN AN INPATIENT COHORT WHO WERE ADMITTED TO A SPECIALIST INPATIENT UNIT}

Abigail Coutinho, Rachel Whitehorn, Emer McKenna. Douglas Macmillan Hospice

10.1136/bmispcare-2019-ASP.183

We aimed to identify whether we are managing our patients pain symptoms effectively using the IPOS as a measuring tool. IPOS forms part of the validated OACC suite of outcomes measures. And also to validate our approach to pain management across all categories and severity of pain and address any deficiencies found. We studied patients over a 3 month period. Interestingly, in the patients with starting higher pain scores $3-4$, we found that $53 \%$ of patients showed improvements in their pain scores, $38 \%$ did not show any 